\title{
Medizinprodukte-Betreiberverordnung - Geltungsbereich, Pflichten und Kosten!
}

\author{
The German „Medical Device Operating Regulation“ (Medizinprodukte- \\ Betreiberverordnung) - Scope, Duties and Costs!
}

\section{Zusammenfassung}

Der deutsche Gesetzgeber hat auch den niedergelassenen Dermatologen mit diversen Pflichten bedacht, was die Anwendung von Medizinprodukten angeht. Hier müssen Bücher geführt werden, Wartungs- und Instandhaltungstätigkeiten durchgeführt werden und zuletzt auch Meldungen an die Behörde gemacht werden. Der Mediziner wird hier zum Handlanger vom Gesetzgeber und ist von Strafen bedroht.

\section{Abstract}

The German legislator regulated the placing of medical devices into the market and even their use. This has effect on the dermatologist who has settled down using his own medical equipment. Documentations are to be kept, actions in maintenance and repair are to be taken and finally reports have to be given to the national authorities. The medical doctor becomes some kind of subworker of the legislator and is threatened by punishment if he refuses this service.
Sicherlich haben die meisten Leser schon von dem „neuen“ Medizinproduktegesetz gehört. Das ist zwar nicht mehr neu, denn immerhin ist es schon seit dem 2. August 1994 veröffentlicht, aber umgesetzt ist das Gesetz mit seinen Verordnungen noch lange nicht überall.

Also lassen Sie uns gemeinsam das Thema angehen und ganz speziell in die Verordnung schauen, die für den Bereich der niedergelassenen Dermatologen interessant sind. Dies ist nur ein kleiner Teil der Gesetzeslage, da das Hauptthema des deutschen Medizinproduktegesetzes die Herstellung von Medizinprodukten ist, was man in der Arztpraxis vermeiden sollte, indem man sich immer strikt an die von den Herstellern mitgelieferte Gebrauchsanweisung der Produkte hält und auch keine Produktkombinationen vornimmt, die von diesen nicht autorisiert wurde.

\section{Welche Produkte in der dermatologischen Praxis fallen unter die Rechtslage?}

Generell kann man sagen, dass alle Produkte, die in der Arztpraxis angewandt werden, um die medizinische Leistung zu erbringen, unter das MPG (Medizinproduktegesetz) oder aber das AMG (Arzneimittelgesetz) bzw. deren Verordnungen fallen. Die schon im August 2002 verabschiedete Medizinprodukte-Betreiberverordnung gilt für alle Medizinprodukte, die in der dermatologischen Praxis angewandt werden. Geregelt wird in der Verordnung das Errichten, Betreiben, Anwenden und Instandhalten von Medizinprodukten aller Art in der täglichen Praxis.

Betreiber im Sinne der Verordnung ist immer derjenige, der Besitzer des Gerätes ist, d.h. der die Sachherrschaft über das Gerät ausübt (s. Böckmann/Frankenberger, Fachwörterbuch, Verlag TÜV Rheinland, Köln). Nach diesem Gesetzeskommentar bedeutet dies, dass selbst bei einem Leasinggerät oder einer Probestel- 
Tab. 1 Beispielhaftes Medizinprodukte-Bestandsverzeichnis für die dermatologische Praxis

\begin{tabular}{|c|c|c|c|c|c|c|c|c|c|}
\hline $\begin{array}{l}\text { Bezeichnung } \\
\text { des Medizin- } \\
\text { produkts }\end{array}$ & $\begin{array}{l}\text { Art und Typ } \\
\text { des Medizin- } \\
\text { produkts }\end{array}$ & $\begin{array}{l}\text { Interne } \\
\text { Ident.-Nr. }\end{array}$ & Serien-Nr. & $\begin{array}{l}\text { UMDNS- } \\
\text { Code }\end{array}$ & $\begin{array}{l}\text { Anschaf- } \\
\text { fungsjahr }\end{array}$ & $\begin{array}{l}\text { Hersteller/ } \\
\text { Importeur }\end{array}$ & $\begin{array}{l}\text { Nummer der } \\
\text { benannten } \\
\text { Stelle }\end{array}$ & Standort & $\begin{array}{l}\text { Fristen STK, } \\
\text { MTK \& } \\
\text { Wartung }\end{array}$ \\
\hline HF-Chirurgie & $\begin{array}{l}\text { Elektrochir- } \\
\text { urgiegeräte }\end{array}$ & 1 & 0123456 & & 2000 & $\begin{array}{l}\text { Dermal } \\
\text { Nonsense Ltd. }\end{array}$ & 0123 & $\mathrm{OP}$ & STK min. 2a \\
\hline $\begin{array}{l}\text { Schleiff- } \\
\text { automat }\end{array}$ & $\begin{array}{l}\text { Elektrochir- } \\
\text { urgiegeräte }\end{array}$ & 2 & 1234567 & & 1998 & $\begin{array}{l}\text { Medical Pro- } \\
\text { dukte GmbH }\end{array}$ & - & OP & Wartung 2a \\
\hline $\begin{array}{l}\text { Dermal- } \\
\text { kamera }\end{array}$ & $\begin{array}{l}\text { Diagnostische } \\
\text { Instrumente/ } \\
\text { Geräte }\end{array}$ & 3 & 2345678 & & 2001 & $\begin{array}{l}\text { Dermal } \\
\text { Nonsense Ltd. }\end{array}$ & 0124 & $\begin{array}{l}\text { Behandlungs- } \\
\text { zimmer }\end{array}$ & MTK 2a \\
\hline $\begin{array}{l}\text { Infrarot- } \\
\text { Fieberthermo- } \\
\text { meter }\end{array}$ & $\begin{array}{l}\text { Diagnostische } \\
\text { Instrumente } \\
\text { und Geräte }\end{array}$ & $5-7$ & 3456789 & & 2003 & $\begin{array}{l}\text { Medical Pro- } \\
\text { dukte GmbH }\end{array}$ & 0125 & $\begin{array}{l}\text { Behandlungs- } \\
\text { zimmer }\end{array}$ & MTK 2a \\
\hline $\begin{array}{l}\text { Blutdruck- } \\
\text { messgerät }\end{array}$ & $\begin{array}{l}\text { Blutdruck- } \\
\text { messgerät }\end{array}$ & 8 & 4567890 & & 1999 & $\begin{array}{l}\text { Medical Pro- } \\
\text { dukte GmbH }\end{array}$ & 0126 & $\begin{array}{l}\text { Behandlungs- } \\
\text { zimmer }\end{array}$ & MTK 2a \\
\hline Absauggerät & $\begin{array}{l}\text { Liposuktion- } \\
\text { Saugsysteme }\end{array}$ & 9 & 5678901 & & 2001 & $\begin{array}{l}\text { Medical Pro- } \\
\text { dukte GmbH }\end{array}$ & 0167 & $\mathrm{OP}$ & $\begin{array}{l}\text { Wartung 1a } \\
\text { MTK 2a }\end{array}$ \\
\hline $\begin{array}{l}\text { Betäubungs- } \\
\text { mittelpumpe }\end{array}$ & $\begin{array}{l}\text { Infusions- } \\
\text { technik }\end{array}$ & 4 & 0112345 & & 2000 & $\begin{array}{l}\text { Medical Pro- } \\
\text { dukte GmbH }\end{array}$ & 0389 & OP & STK min. 2a \\
\hline OP-Leuchte & $\begin{array}{l}\text { Medizinische } \\
\text { Leuchten }\end{array}$ & $10+11$ & 6789012 & & 2002 & $\begin{array}{l}\text { Medical Pro- } \\
\text { dukte GmbH }\end{array}$ & - & OP & Wartung 1a \\
\hline Ultraschallbad & $\begin{array}{l}\text { Sterilisation u. } \\
\text { Desinfektion }\end{array}$ & 12 & 7890123 & & 1997 & $\begin{array}{l}\text { Dermal } \\
\text { Nonsense Ltd. }\end{array}$ & 0545 & Aufbereitung & $\begin{array}{l}\text { Wartung/ } \\
\text { Validierung 1a }\end{array}$ \\
\hline $\begin{array}{l}\text { Dampf- } \\
\text { sterilisator }\end{array}$ & $\begin{array}{l}\text { Sterilisation u. } \\
\text { Desinfektion }\end{array}$ & 13 & 8901234 & & 1997 & $\begin{array}{l}\text { Medical Pro- } \\
\text { dukte GmbH }\end{array}$ & 0357 & Aufbereitung & $\begin{array}{l}\text { Wartung/ } \\
\text { Validierung 1a }\end{array}$ \\
\hline $\begin{array}{l}\text { Patienten- } \\
\text { datenbank } \\
\text { mit Bildbear- } \\
\text { beitung }\end{array}$ & $\begin{array}{l}\text { Medizinische } \\
\text { Dokumenta- } \\
\text { tionssysteme }\end{array}$ & 14 & 9012345 & & 2003 & $\begin{array}{l}\text { Dermal } \\
\text { Nonsense Ltd. }\end{array}$ & 0569 & $\begin{array}{l}\text { Behandlungs- } \\
\text { zimmer }\end{array}$ & MTK 2a \\
\hline
\end{tabular}

lung eines Produktes durch den Hersteller die Betreiberpflichten immer bei dem niedergelassenen Mediziner liegen, obwohl der Hersteller oder Händler weiterhin das Eigentum an dem Produkt hält.

\section{Pflichten aus der Betreiberverordnung}

Die deutsche Medizinprodukte-Betreiberverordnung kennt wie die gesamte Rechtslage auch verschiedene Produktkategorien. Diese Produktunterschiede resultieren dann auch in Betreiberpflichten, die für den niedergelassenen Mediziner unterschiedlich sind in Aufwand und Kostenintensität.

Grundsätzlich gilt, dass der Anwender sich vor jeder Anwendung aller in der Arztpraxis angewandten Medizinprodukte von der ausreichenden Produktsicherheit überzeugen muss. Auch muss der Arzt dafür sorgen, dass lediglich Mitarbeiter mit der für die Anwendung der Produkte notwendigen Kenntnisse und Fähigkeiten mit deren Anwendung betraut werden. Zudem ist es Pflicht des Mediziners dafür zu sorgen, dass das Produkt lediglich entsprechend der vom Hersteller vorgesehenen Anwendung eingesetzt wird. Diese ist in der Gebrauchsanweisung beschrieben. Daher ist leicht verständlich, warum die Gebrauchsanweisung der Produkte bzw. Geräte bei den Anwendern jederzeit während der Anwendung greifbar und natürlich auch gut bekannt sein muss.
Der niedergelassene Arzt, also der Betreiber im Sinne der Rechtslage, hat für alle aktiven nichtimplantierbaren Medizinprodukte der jeweiligen Betriebsstätte (hier also der Arztpraxis) ein Bestandsverzeichnis zu führen. Grob und etwas vereinfacht gesagt bedeutet das, dass alle elektrisch betriebenen Geräte in einer stets aktuell gehaltenen Liste aufgeführt werden müssen.

In dieser Liste müssen ebenfalls die Wartungs-, Instandhaltungsund Prüfintervalle geführt werden, so jedenfalls definiert es die Verordnung. Sehen Sie hierzu das Beispiel in Tab.1, das ein solches Bestandsverzeichnis mit typischen Produkten in der dermatologischen Praxis aufzeigt.

Die notwendigen Wartungs- und Prüftätigkeiten sowie die zugehörigen Intervalle sind den Gebrauchsanweisungen der Hersteller zu entnehmen. Jedoch gelten diese Vorgaben der Hersteller nur, solange die Erfahrungen der Anwender nicht einen höheren Wartungsaufwand für die individuelle Nutzung der Produkte in der jeweiligen Arztpraxis empfehlen. Diese eventuell nötige zusätzliche Wartungs- und Instandhaltungstätigkeit muss natürlich entsprechend begründet, beschrieben und geplant werden. Alle diese Arbeiten müssen von entsprechend befähigten Mitarbeitern oder aber Fremdunternehmen durchgeführt werden, wobei der niedergelassene Arzt als Betreiber wiederum nachweispflichtig bezüglich der Kompetenz, Befähigung und Ausstattung ist. 
Über die Wartungs- und Instandhaltungstätigkeiten hinaus werden für alle Produkte mit Messfunktion regelmäßige messtechnische Kontrollen (MTK) gefordert. Für andere gesondert in der Anlage 1 der Medizinprodukte-Betreiberverordnung aufgeführten Medizinprodukte muss eine regelmäßige sicherheitstechnische Kontrolle (STK) entsprechend den allgemein anerkannten Regeln der Technik (also z. B. entsprechend harmonisierter Normen) in den vom Hersteller angegebenen Fristen durchgeführt werden. Die MTKs und STKs sind mindestens alle zwei Jahre von entsprechend befähigten Mitarbeitern oder Unternehmen durchzuführen, wie wir dies bereits von den Wartungs- und Instandhaltungsarbeiten kennen.

Dokumentiert werden muss dies alles in dem „Medizinproduktebuch“, das auch für die zusätzlich in der Anlage 2 benannten Medizinprodukte von den Dermatologen als Betreiber geführt werden muss.

Betroffen von diesen Regelungen zu den sicherheitstechnischen Kontrollen (STK) sind z. B. HF-chirurgische Geräte oder Behandlungslaser, während Absauggeräte, Dermalkameras oder aber triviale Blutdruckmessgeräte einer regelmäßigen messtechnischen Kontrolle bedürfen.

In der Betreiberverordnung nicht explizit geregelt sind Prüfungen zu Produkten wie z.B. Sterilisatoren oder Ultraschallbäder zur Desinfektion. Dennoch ist dies kein Grund zur übermäßigen Freude, denn auch diese Geräte müssen entsprechend des Standes der Technik regelmäßig gewartet und vor allem bezüglich ihrer hygienischen Wirkung revalidiert werden. Hierfür gibt es wiederum technische Regeln bzw. Normen, die einzuhalten sind und deren Einhaltung nachzuweisen ist. Darüber hinaus ist zu jeder durchgeführten Sterilisation auch eine spezielle Dokumentation anzufertigen und aufzubewahren. Doch dies kennen Sie sicherlich, da diese Regelungen nun schon viele lange Jahre gültig sind.

\section{Meldepflichten}

In dem bereits erwähnten Medizinproduktebuch sollen auch Aufzeichnungen über aufgetretene Funktionsstörungen der Medizinprodukte und wiederholte gleichartige Bedienungsfehler in der Nutzung geführt werden. Neben dieser eher für den internen Gebrauch gedachten Dokumentation sind die Dermatologen wie alle niedergelassenen Mediziner, Krankenhäuser und Pflegeeinrichtungen verpflichtet, Vorkommnisse mit den Medizinprodukten an die zuständige Bundesoberbehörde (BfArM, Bundesinstitut für Arzneimittel und Medizinprodukte, Bonn) zu melden. Diese Meldungen haben unverzüglich zu erfolgen, nachdem der Betreiber Kenntnis von dem Auftreten des Vorkommnisses erhalten hat.

$\mathrm{Zu}$ melden ist dabei jede Funktionsstörung, jeder Ausfall oder Änderung der Merkmale oder der Leistung oder jede Unsachgemäßheit der Kennzeichnung oder der Gebrauchsanweisung eines Medizinprodukts, die unmittelbar oder mittelbar zum Tod oder zu einer schwerwiegenden Verschlechterung des Gesund-

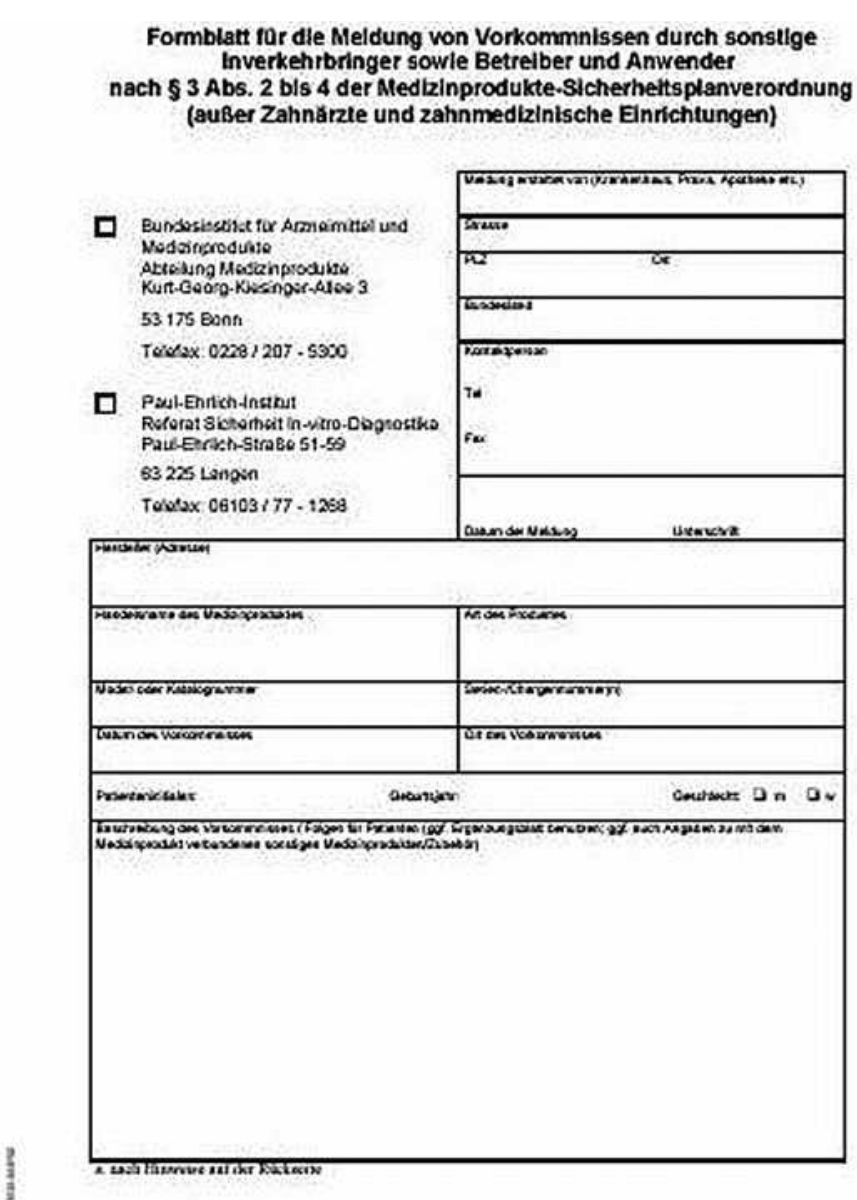

Abb. 1 Meldeformular der DIMDI für Vorkommnisse mit Medizinprodukten in der Arztpraxis

heitszustands eines Patienten, eines Anwenders oder einer anderen Person geführt hat, geführt haben könnte oder führen könnte (nach §2 Begriffsbestimmungen, MPSV).

Also muss jeder Dermatologe eine schriftliche Meldung an das BfArM schicken, wenn eines seiner Geräte sich im Gebrauch als nicht sicher erweist, oder aber die Gebrauchsanweisung so verfasst ist, dass Mitarbeiter sie falsch interpretieren können, woraus eine Gefährdung der Patienten, Anwender oder auch anderer Praxisbesucher resultieren könnte.

Für die Meldung dieser Vorkommnisse werden auf dem Internet Formulare unter der Adresse www.dimdi.de bereitgestellt, die von dem Betreiber entsprechend ausgefüllt und der zuständigen Behörde umgehend zugestellt werden müssen (Abb.1).

\section{Kontrolle und Kosten}

Den meisten unter Ihnen wird bekannt sein, dass es auch in der Vergangenheit eine Rechtslage gab, die ähnliches wie das oben beschriebene regelte. Diese Rechtsvorschrift nannte sich MedGV (Medizingeräteverordnung) und erfreute sich, zumindest im niedergelassenen Bereich, eines nur geringen Erfüllungsgrades. Warum also soll jetzt mit der neuen Rechtslage alles ganz anders sein? 
Warum nicht auch dieses Mal darauf hoffen, dass die Tinte auf den von Ihnen geforderten Dokumenten immer gerade noch trocken wird, bevor die Behörde kontrollieren kommt? - Nun das Netz um die Betreiber ist enger geworden und die Eigenverantwortlichkeit der betroffenen Mediziner wurde m.E. in der Rechtslage deutlich erhöht. Dies bedeutet, dass bei einer Schädigung von Patienten oder Dritten in Zukunft öfter die Frage an den Arzt gestellt wird, wie sicher er sein konnte, dass das Gerät sicher funktioniert! Dies dürfte bedeuten, dass das Deuten auf den Hersteller nur dann noch ausreichend Haftungssicherheit verspricht, wenn dieser mit den notwendigen Wartungs-, Instandhaltungs- und Prüfungspflichten beauftragt wurde.

Zudem werden Sie in Gesprächen mit Ihren Kollegen bemerkt haben, dass die verschiedenen Behörden Ihre Kontrollpflichten auch gegenüber dem Ärztestand in der jüngsten Vergangenheit immer öfter wahrnehmen.

Wie also alle diese Anforderungen erfüllen? Sicherlich sind Sie mit mir einer Meinung, dass die Wartungs- und Instandhaltungstätigkeiten über die ärztliche Leistung deutlich hinausgehen und damit deren Wahrnehmung durch Sie oder Ihr Team Ihre berufliche Effektivität empfindlich mindern würde. Also benötigen wir dafür Dienstleister.

Nicht immer haben Sie und Ihre Kollegen allerdings mit einem „Werkskundendienst“ zur Werterhaltung Ihrer Geräte die besten Erfahrungen gemacht. Scheint doch der günstige Anschaffungspreis des einen oder anderen Geräts über die später benötigten Ersatzteile bzw. Kundendienstpreise finanziert zu werden.

Der Aufbau einer eigenen Service-Crew wird auch nur in den wenigsten Fällen für eine dermatologische Arztpraxis betriebswirtschaftlich interessant sein. Also muss der freie Markt der Dienstleistungsunternehmen nach entsprechenden qualifizierten Unternehmen untersucht werden. Zweck dieser Marktuntersuchung sollte immer sein, die Kosten für die einzelne Arztpraxis bei hoher Dienstleistungsqualität niedrig zu halten. Dieser Aufwand für Wartung, Instandhaltung und Prüfung sollte schon bei einer Neubeschaffung von Medizinprodukten kalkulierbar und bekannt sein, da nur so eine langfristig betriebswirtschaftlich sinnvolle Beschaffungsentscheidung durch den Mediziner erfolgen kann.

\section{Wer soll das alles machen?}

Alles vorangegangen Gesagte macht klar, dass die Erfüllung der gesetzlichen Pflichten durchaus zeitintensiv ist und auch eine entsprechende Qualifikation voraussetzt. Daher wird auch ein entsprechender Dienstleister sich diese mit einiger Verantwortung verbundene Leistung gut bezahlen lassen.

Stellt jetzt jeder niedergelassene Dermatologe sich selbst der Aufgabe eine Marktanalyse anzustellen und die Qualität, Leistung und Preise der Dienstleistungsunternehmen abzuschätzen, geht dafür enorm viel Zeit und auch Geld ins Land. Auch wird sich sicherlich jeder verantwortungsbewusste Praxisinhaber nach dieser Analyse fragen, ob er nun alle Aspekte dieser und eventuell anderer geltenden Rechtslagen sicher erfüllt hat.

Daher hat sich die schon oft beschriebene Qualitätsinitiative „Darmstädter Modell“ bereits diese Aufgabe vorgenommen, die Sie bei der sinnvollen Auswahl von Dienstleistungsunternehmen zur Erfüllung der gesetzlichen Pflichten unterstützt.

Die vom Darmstädter Modell erzeugte Marktübersicht stützt sich auf eine Bewertung des Unternehmens und seiner angebotenen Dienstleistungen entsprechend des Forderungskatalogs innerhalb des Qualitätsmanagement-Modells. Alle entsprechenden Unternehmen müssen sich einer regelmäßig wiederkehrenden Bewertung unterziehen.

Damit wird gewährleistet sein, dass der am Darmstädter Modell teilnehmende niedergelassene Mediziner für nur einen geringen finanziellen Beitrag Zugriff auf arbeitsintensive Informationen erhält, die wiederum eine Kostenersparnis bei hoher Qualität bewirkt.

Zudem kann dieses System durch die relative Vergleichbarkeit der Aufträge an die Dienstleitungsorganisationen zu einer entsprechenden Standardisierung führen, die wiederum einen Preisvorteil (Mengenrabatt), aber auch eine höhere Durchführungssicherheit erzeugt.

Ein weiterer netter Nebeneffekt kann die Datensammlung durch das Darmstädter Modell sein. Hierdurch kann durch die gesammelten Erfahrungen aller teilnehmenden Arztpraxen eine Markttransparenz bezüglich Dienstleistungsqualität, aber auch Produktqualität und Anwendungssicherheit erzeugt werden. Diese Daten können dann in die nötigen zukünftigen Anschaffungsentscheidungen einfließen und unnötige Kosten vermeiden.

Weitere Informationen erhalten Sie beim Autor und unter www.consultingandmore.com. 